\title{
The Image Gently ALARA CT summit on new CT technologies for children
}

\author{
Marilyn J. Goske • Keith J. Strauss • Sjirk J. Westra • \\ Donald P. Frush
}

Received: 28 April 2014 / Accepted: 1 June 2014

(C) Springer-Verlag Berlin Heidelberg 2014

Under the leadership of Drs. Marilyn J. Goske, Sjirk J. Westra and Donald P. Frush and Mr. Keith J. Strauss, and with the administrative assistance of Ms. Angela Davis, the Alliance for Radiation Safety in Pediatric Imaging and the Society for Pediatric Radiology organized the third ALARA CT meeting and second Image Gently CT meeting into a single conference, titled "The Image Gently ALARA CT Summit: How to Use New CT Technologies for Children." The meeting, conducted in Orlando, FL, on Feb. 21-22, featured 21 course faculty members (including 9 pediatric radiologists, 2 adult radiologists, 8 medical imaging physicists, a pediatrician and a radiologic technologist), who collectively presented 30 lectures.

Highlights of the meeting included four non-commercial rapid-fire presentations that paired pediatric radiologists with medical imaging physicists to discuss the advantages and disadvantages of the four major CT vendors' equipment. More than 140 attendees filled the conference room at the Hyatt Regency Orlando International Airport hotel. Conference presentations were divided into sections covering CT scanner operations, patient dose and potential risks, management of patient dose, quality improvement, and training and communication.

The meeting provided an overview about why and when to use CT in children from a radiologist's perspective and was complemented by a pediatric emergency room physician's view.

M. J. Goske $(\bowtie) \cdot$ K. J. Strauss

Department of Radiology,

Cincinnati Children's Hospital Medical Center,

3333 Burnet Ave., Cincinnati, OH 45229-3039, USA

e-mail: Marilyn.Goske@cchmc.org

\section{S. J. Westra}

Section of Pediatric Radiology, Massachusetts General Hospital,

Boston, MA, USA

D. P. Frush

Division of Pediatric Radiology, Duke University Medical Center, Durham, NC, USA
The meeting paid particular attention to CT scanner operations. Detailed lectures on automatic exposure control and iterative reconstruction were included. The conference was rounded out with a discussion of the challenges in the scientific field resulting from the lack of standardized nomenclature.

Two sessions were dedicated to the complexity of radiation dose, and updates were provided on the latest understanding about radiation risk. A point-counterpoint session presented the challenges of pediatric protocol development and compared scanner-specific protocols with universal protocols. The concept of optimizing image quality in relation to $\mathrm{CT}$ dose was discussed. Individual dose tracking was also presented. The audience was introduced to the American College of Radiology's adult and pediatric dose index registries as a tool for quality improvement in their practice.

One session reviewed practical approaches to implementing quality improvement into pediatric CT practice. The last day's formal session presented the art of communication with parents and referring providers. By including a pediatric emergency room specialist, the meeting emphasized the importance of communication among pediatric specialists in the care of children. The meeting was highly interactive, with robust questions from the audience after each session.

The "Image Gently ALARA CT Summit: How to Use New CT Technologies for Children" provided an exciting and informative forum for the audience to interact with the faculty and collect in-depth information about current topics related to radiation safety as it applies to the practice of pediatric CT.

This peer-reviewed supplement to the journal Pediatric Radiology includes a summary of the presentations as review articles. It is our hope that this supplement will allow the content of the meeting to reach a broad audience and will benefit the readership and members of the Society for Pediatric Radiology and beyond.

Conflicts of interest None 